\title{
Dehiscence of aortic valve commissure complicated by aortic regurgitation
}

\author{
Toshihiro Fukui, MD, Tomoki Shimokawa, MD, Ken-u Fumimoto, MD, Susumu Mananbe, MD, Naomi Ozawa, MD, \\ and Shuichiro Takanashi, MD, Tokyo, Japan
}

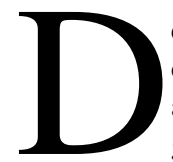

ehiscence or aortic valve commissural tear is a rare cause of acute aortic regurgitation (AR). We report a case of aortic valve commissural dehiscence with progressive AR and aortic root dilatation that was successfully repaired with aortic root and ascending aortic replacements.

\section{Clinical Summary}

A 67-year-old man was admitted to the Sakakibara Heart Institute with new onset of exertional precordial pain. His electrocardiogram and cardiac enzymes were normal. Contrast-enhanced computed tomography showed a small pericardial effusion but did not reveal aortic dissection. A transthoracic echocardiogram showed mild AR with no dilatation of the cardiac chamber. Furthermore, a flap or intimal tear of the proximal aorta was absent on transthoracic echocardiography. Because the patient had a history of paroxysmal atrial fibrillation, an antiarrhythmic drug was prescribed before discharge. After 1 month, he was readmitted to the Sakakibara Heart Institute because of the progression of dyspnea on exertion. $\mathrm{He}$ had a new diastolic murmur and a systolic murmur. Chest x-rays revealed pulmonary congestion. Transthoracic echocardiography demonstrated severe AR and moderate mitral regurgitation with dilatation of the left ventricle. Computed tomography showed aneurysmal enlargement of the ascending aorta with aortic root dilatation (ascending aorta, $47 \mathrm{~mm}$; sinotubular junction, $44 \mathrm{~mm}$; sinus of Valsalva, $44 \mathrm{~mm}$ ); however, there was no evidence of aortic dissection. Transesophageal echocardiography was performed to assess the cause of AR. Transesophageal echocardiography revealed a prolapse of both the right and left coronary cusps (Figure 1) with no intimal flap in the ascending aorta. Surgery was planned after medical control of the patient's heart failure.

A median sternotomy was performed. When the pericardium was opened, bloody serous effusion was observed. Cardiopulmonary bypass was performed with ascending aortic and bicaval venous cannulations. Bilateral pulmonary vein isolation with radiofrequency ablation was done before cardiac arrest. After aortic clamp and cardiac arrest, mitral annuloplasty with a semi-rigid ring (size, $28 \mathrm{~mm}$ )

\footnotetext{
From the Department of Cardiovascular Surgery, Sakakibara Heart Institute, Tokyo, Japan.

Received for publication Jan 4, 2008; accepted for publication Jan 15, 2008.

Address for reprints: Toshihiro Fukui, MD, Department of Cardiovascular Surgery, Sakakibara Heart Institute, 3-16-1 Asahi-cho, Fuchu City, Tokyo 183-0003, Japan (E-mail: tfukui-cvs@umin.ac.jp).

J Thorac Cardiovasc Surg 2008;135:1178-9

$0022-5223 / \$ 34.00$

Copyright $\odot 2008$ by The American Association for Thoracic Surgery doi:10.1016/j.jtcvs.2008.01.011
}

was performed. The body temperature was decreased to $25^{\circ} \mathrm{C}$, systemic perfusion was temporarily arrested, and retrograde cerebral perfusion was started. The ascending aorta was resected and replaced with a 24-mm knitted Dacron graft (Hemashield Gold, Boston Scientific, Natick, Mass) with 1 branch. After the completion of distal aortic anastomosis, systemic perfusion through the side branch of the graft was resumed. There was no dissection or intramural hematoma in the ascending aorta. There was a dehiscence of the commissure between the right and left coronary cusps of the aortic valve (Figure 2). The valve leaflets were slightly atherosclerotic but almost intact, and the aortic wall was normal. We performed aortic root replacement with a commercially available composite valved conduit (23-26 mm; Carboseal, CarboMedics, Austin, Tex). Total cardiopulmonary bypass time was 188 minutes. The postoperative course was uneventful, and no neurologic deficit occurred.

\section{Discussion}

Acute AR sometimes occurs as a complication of acute aortic dissection. Movsowitz and colleagues ${ }^{1}$ reported the causative mechanisms of AR using transesophageal echocardiography, including dilatation of a sinotubular junction, aortic leaflet prolapse, and dissection flap prolapse through the aortic valve. Aortic leaflet prolapse occurs when dissection extends into the aortic root and disrupts the normal leaflet attachments to the aortic wall. However, in the present case, prolapse of the right and left aortic leaflets occurred by a dehiscence of the commissure between the right and left aortic cusps. This is a rare condition and has been documented in some case reports. ${ }^{2-5}$ Although the cause of aortic commissural dehiscence is unknown, hypertension is considered to be the most probable cause. ${ }^{5}$ However, in our patient, hypertension was not observed before admission.

Surgical treatments for dehiscence of aortic valve commissures include repair of the commissure with a valve, replacement of the aortic valve, and aortic root replacement. In the literature, aortic valve replacement is the common procedure. ${ }^{2,3,5}$ Sakakibara and colleagues $^{3}$ reported that aortic valve replacement with fixation of the dehiscent aortic wall should be the treatment of choice. Aortic root replacement has been performed in some reports. ${ }^{2,4}$ We performed aortic root replacement because the aortic root and ascending aorta were dilated. Aortic root replacement with a valved conduit is an established and straightforward procedure. The aortic valve-sparing operation was not attempted in the present case because other cardiac procedures (mitral valve annuloplasty, ascending aortic replacement, and radiofrequency ablation for pulmonary vein isolation) were necessary.

\section{Conclusions}

Dehiscence of aortic valve commissure is a rare cause of acute AR. The present case report also suggests that continuous care 


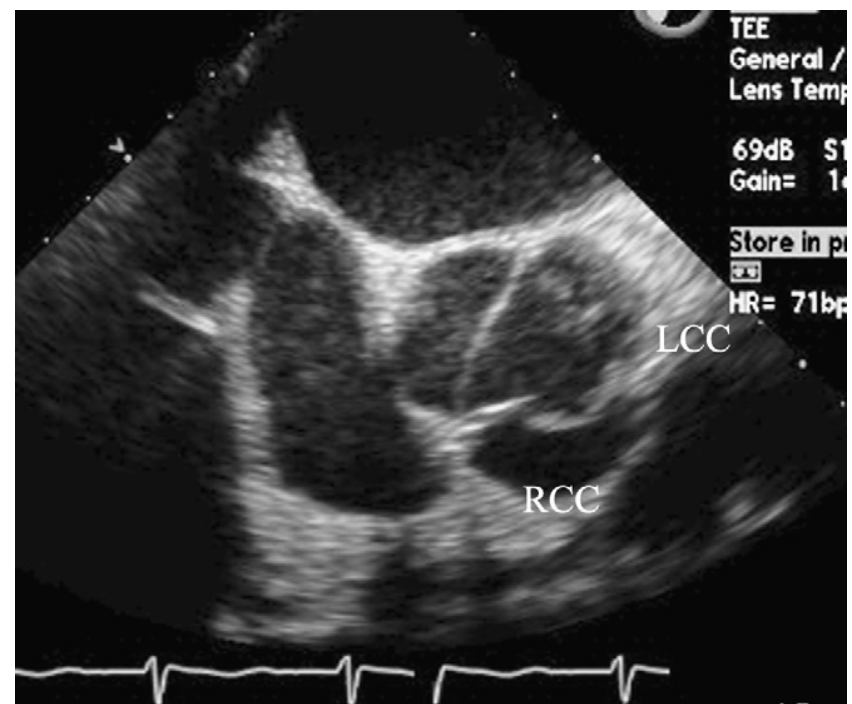

Figure 1. Transesophageal echocardiogram demonstrating prolapse of both the right and left coronary cusps with no flap in the ascending aorta. TEE, Transesophageal echocardiography; $H R$, heart rate; $L C C$, left coronary cusp; $R C C$, right coronary cusp.

must be taken even if initial examinations with echocardiography or computed tomography do not reveal aortic valve commissural tear because gradual worsening of AR may occur in such patients.

\section{References}

1. Movsowitz HD, Levine RA, Hilgenberg AD, Isselbacher EM. Transesophageal echocardiographic description of the mechanisms of aortic regurgitation in acute type A aortic dissection: implications for aortic valve repair. J Am Coll Cardiol. 2000;36:884-90.

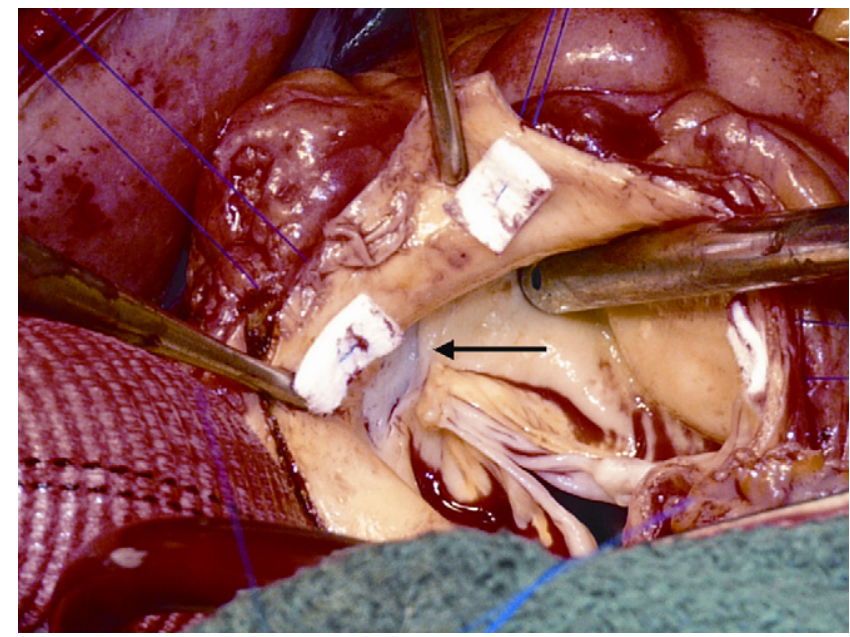

Figure 2. Intraoperative photograph: local dehiscence of the aortic commissure between the right and left coronary cusps of the aortic valve. Local dehiscence (arrow).

2. Aoyagi S, Fukunaga S, Oishi K. Aortic regurgitation due to non-traumatic rupture of the aortic valve commissures: report of two cases. J Heart Valve Dis. 1995;4:99-102.

3. Sakakibara Y, Gomi S, Mihara W, Mitsui T, Unno H, Doi T. Acute heart failure due to local dehiscence of aortic wall at aortic valvular commissure. Jpn J Thorac Cardiovasc Surg. 2000;48:80-2.

4. Kupersmith AC, Belkin RN, McClung JA, Moggio RA. Aortic valve commissural tear mimicking type A aortic dissection. J Am Soc Echocardiogr. 2002;15:658-60.

5. Newcomb AE, Rowland MA. Nontraumatic localized dehiscence of the proximal ascending aorta through an aortic valve commissure. Ann Thorac Surg. 2004;78:321-3. 